\title{
Caracterización química de pigmentos cerámicos a base de sulfoseleniuro de cadmio
}

\author{
M. F. GAZULLA BARREDA; M. RODRIGO EDO; E. BLASCO ROCA; M. ORDUÑA CORDERO \\ Instituto de Tecnología Cerámica. \\ Asociación de Investigación de las Industrias Cerámicas. \\ Universitat Jaume I. Castellón. España.
}

\begin{abstract}
El objetivo del presente trabajo es el desarrollo de una metodología que permita la caracterización química completa de pigmentos cerámicos a base de sulfoseleniuro de cadmio, incluyendo elementos mayoritarios y minoritarios.

Para desarrollar la metodología, se seleccionaron cinco pigmentos a base de sulfoseleniuro de cadmio, estudiando las distintas fases del proceso de medida, desde la preparación de la muestra hasta la optimización de la medida de los distintos componentes de los pigmentos por técnicas espectroscópicas (WD-FRX y análisis elemental por combustión y detección mediante IR).

El método de caracterización química desarrollado se validó con patrones sintéticos preparados a partir de la mezcla de materiales de referencia certificados y óxidos puros debido a que no existen comercialmente materiales de referencia certificados de este tipo de pigmentos. Este método se puede utilizar para la caracterización química completa de pigmentos a base de sulfoseleniuro de cadmio con incertidumbres muy bajas para todos los elementos analizados.
\end{abstract}

Palabras clave: pigmentos de sulfoseleniuro de cadmio, propiedades quimicas, espectroscopía (WD-FRX), análisis elemental (combustión $y$ detección mediante IR).

\section{Chemical characterisation of zircon-cadmium sulfoselenide ceramic pigments}

The present paper addresses the development of a methodology that allows the complete chemical characterisation of zirconcadmium sulfoselenide ceramic pigments including minor and major elements.

To develop the methodology, five zircon-cadmium sulfoselenide pigments with different hues were selected, studying the different measurement process steps, from sample preparation to the optimisation of the measurement of the different components of the pigments by spectroscopic techniques (WD-XRF and elemental analysis by combustion and IR detection).

The chemical characterisation method developed was validated with synthetic standards prepared from the mixture of certified reference materials and pure oxides because no certified referenced materials of this type of pigments were commercially available. The developed method can be used for a complete chemical characterization of zircon-cadmium sulfoselenide ceramic pigments with a very low uncertainty for all the elements analysed.

Keywords: zircon-cadmium sulfoselenide pigment, chemical properties, spectroscopy (WD-XRF), elemental analysis (combustion infrared detection).

\section{INTRODUCCIÓN}

Los pigmentos a base de sulfoseleniuro de cadmio $\left(\mathrm{ZrSiO}_{4}-\right.$ $\mathrm{CdS}_{x} \mathrm{Se}_{1-\mathrm{x}}$ ) son un tipo de pigmentos que se usa en las industrias cerámica y del plástico y que genera una amplia paleta de colores, desde el amarillo hasta el rojo y granate, dependiendo de la proporción de selenio $(1,2)$.

Existen distintos estudios sobre la formación y síntesis de este tipo de pigmentos; por ejemplo, se estudia la cinética de formación del pigmento y se utiliza difracción de rayos $X$ (DRX) y espectroscopía de ultravioleta visible para analizarlo cualitativamente (3). En otros trabajos se estudia el proceso de encapsulación para que sean más estables (4-5), pero mayoritariamente estos estudios están focalizados en nuevos métodos de síntesis para obtener pigmentos térmicamente resistentes, como el uso de cristales mixtos de $\mathrm{CdO}$ y CdSe en lugar de un ión dopante, etc.; y usando técnicas tales como DRX, microscopía electrónica de transmisión (MET) o espectrometría de infrarrojo (IR) para caracterizar el producto final (4), pero ninguno de ellos describe una metodología para su caracterización química cuantitativa , lo cual es de especial relevancia para disponer de una completa caracterización del pigmento.

Estos materiales presentan problemas considerables para su análisis químico debido a que contienen componentes de naturaleza muy distinta y algunos de ellos se volatilizan durante el proceso de preparación de la muestra para la medida.

Este trabajo se llevó a cabo para establecer un método cuantitativo de caracterización de pigmentos a base de 
sulfoseleniuro de cadmio utilizando espectrometría de fluorescencia de rayos $\mathrm{X}$ por dispersión de longitudes de onda (WD-FRX) y análisis elemental por combustión y detección mediante IR.

\section{PARTE EXPERIMENTAL}

\subsection{Selección de los pigmentos a base de sulfoseleniuro de cadmio}

Se seleccionaron cinco muestras industriales de pigmentos de sulfoseleniuro de cadmio referenciadas como: Pigmento 1, Pigmento 2, Pigmento 3, Pigmento 4 y Pigmento 5; para ser caracterizadas químicamente. Estas muestras se analizaron previamente mediante difracción de rayos $X(D R X)$ de la muestra en polvo, mediante un difractómetro BRUKER ThetaTheta modelo D8 Advance y utilizando los archivos JCPDS para fases cristalinas puras. Todos los pigmentos presentaban como fase cristalina mayoritaria Circón $\left(\mathrm{ZrSiO}_{4}\right)$ y como minoritarias las siguientes fases: Cuarzo $\left(\mathrm{SiO}_{2}\right)$, Baddeleyita $\left(\mathrm{ZrO}_{2}\right)$, Tazheranita $\left(\mathrm{ZrO}_{2}\right)$ y sulfoseleniuro de cadmio $\left(\mathrm{Cd}_{2} \mathrm{SSe}\right)$.Los pigmentos 3 y 5 presentaban además sulfuro de selenio $\left(\mathrm{SeS}_{2}\right)$, mientras el Pigmento 1 presentaba seleniuro de cadmio $\left(\beta-\mathrm{CdSe} \mathrm{e}_{2}\right)$.

\subsection{Procedimiento experimental}

El método desarrollado está basado en la determinación de azufre mediante combustión y detección mediante IR y el resto de elementos mediante WD-FRX, preparando la muestra en forma de pastillas prensadas.

\subsubsection{DETERMINACIÓN MEDIANTE WD-FRX}

Inicialmente, la muestra se preparó en forma de pastillas prensadas y discos fundidos (perlas) para el análisis semicuantitativo de los elementos presentes en los pigmentos seleccionados, utilizando el programa UNIQUANT suministrado por la firma PANalytical, basado en parámetros fundamentales. Las medidas se llevaron a cabo utilizando un espectrómetro de WD-FRX modelo AXIOS de la firma PANalytical provisto de un tubo de rodio, con una potencia de $4 \mathrm{~kW}$ y tres detectores: flujo, centelleo y sellado. En los resultados obtenidos se observó que algunos elementos de las muestras se volatilizaban durante el proceso de preparación de las perlas. Estos elementos eran Se y S. En un estudio previo (6), se puso a punto una metodología para el análisis de azufre en muestras geológicas preparando la muestra en forma de perlas. En dicho trabajo, se eliminaba la volatilización del azufre mediante la adición de óxido de bario y nitrato sódico para formar un compuesto de azufre estable $\left(\mathrm{BaSO}_{4}\right)$. Se realizaron distintas pruebas con los pigmentos de sulfoseleniuro, pero no se consiguió eliminar la volatilización del azufre en este tipo de materiales. Por tanto, la preparación de muestra para la medida mediante WD-FRX se debía realizar preparando la muestra en forma de pastillas.

\subsubsection{Preparación de pastillas prensadas}

Las muestras presentaban una distribución granulométrica con un $99 \%$ de las partículas por debajo de $60 \mu \mathrm{m}$. Se prepararon pastillas prensadas de $32 \mathrm{~mm}$ de diámetro pesando $9.0000 \mathrm{~g}$ de muestra, añadiendo $2.5 \mathrm{ml}$ de una disolución de n-butil metacrilato al $13.7 \%$ en acetona, mezclándolo en un mortero de ágata y prensando a una presión de $100 \mathrm{kN}$ en una prensa hidraúlica de la firma CASMON (7).

\subsubsection{Materiales utilizados para la calibración y la validación de las medidas}

Dado que no existen materiales de referencia certificados de pigmentos a base de sulfoseleniuro de cadmio, se prepararon patrones sintéticos para la validación y calibración a partir de la mezcla de distintos materiales de referencia certificados y óxidos puros en las proporciones adecuadas para obtener la composición deseada.

La calibración y validación de las medidas mediante WD-FRX se llevaron a cabo con los siguientes materiales de referencia y óxidos puros.

a. Materiales de referencia certificados y reactivos químicos utilizados en la preparación de patrones sintéticos para la calibración: BCS-CRM 313/1 High Purity Silica, BCS-RM No 358 Zirconia, SARM 13 Zirconium concentrate, SeS $_{2}$ de Alfa Aesar y CdS de Merck.

b. Materiales de referencia certificados y reactivos químicos utilizados para la preparación de patrones sintéticos para la validación: BCS-CRM 388 Zircon, CdS de Aldrich, CdO de Fluka y $\mathrm{SeS}_{2}$ de Aldrich.

\subsubsection{Selección de las condiciones de medidas}

Se optimizaron las condiciones de medida (línea analítica, detector, cristal, potencia del tubo, ángulo, fondos, PHDs (distribución de altura de impulsos) y tiempo de medida) para cada elemento. En la tabla 1 se muestran las condiciones de medida óptimas para cada elemento.

\subsubsection{Calibración}

Se prepararon 6 patrones sintéticos a partir de la mezcla de los materiales de referencia certificados especificados en

TABLA 1. CONDICIONES DE MEDIDA MEDiANTE WD-FRX

\begin{tabular}{|c|c|c|c|c|c|c|c|c|c|c|c|}
\hline Elemento & Línea & Cristal & Detector & $\begin{array}{c}\text { Voltaje } \\
(\mathrm{kV})\end{array}$ & $\begin{array}{l}\text { Intensidad } \\
\quad(\mathrm{mA})\end{array}$ & $\begin{array}{l}\text { Ángulo } \\
(2 \theta)\left({ }^{\circ}\right)\end{array}$ & Fondo 1 & Fondo 2 & PHD LL & PHD UL & $t(s)$ \\
\hline $\mathrm{Zr}$ & $\mathrm{K}_{\alpha}$ & LiF220 & Centelleo & 30 & 30 & 32.0446 & -0.41 & 0.52 & 28 & 70 & 30 \\
\hline $\mathrm{Si}$ & $\mathrm{K}_{\alpha}$ & InSb 111 & Flujo & 30 & 100 & 144.7988 & -1.29 & 0.98 & 30 & 70 & 40 \\
\hline $\mathrm{Al}$ & $\mathrm{K}_{\alpha}$ & PE 002 & Centelleo & 30 & 100 & 144.9142 & -0.88 & 1.76 & 29 & 29 & 30 \\
\hline $\mathrm{Hf}$ & $\mathrm{L}_{\beta 1}$ & LiF 200 & Centelleo & 60 & 50 & 39.8880 & -0.37 & 0.39 & 25 & 72 & 30 \\
\hline $\mathrm{Ti}$ & $\mathrm{K}_{\alpha}$ & LiF 200 & Flujo & 30 & 90 & 86.1686 & -0.47 & 0.54 & 34 & 66 & 30 \\
\hline $\mathrm{S}$ & $\mathrm{K}_{\alpha}$ & Ge 111 & Flujo & 30 & 100 & 110.7756 & -0.77 & 1.30 & 34 & 69 & 50 \\
\hline Se & $\mathrm{K}_{\alpha}$ & LiF 200 & Flujo & 60 & 50 & 31.8546 & -0.46 & 0.47 & 26 & 73 & 50 \\
\hline $\mathrm{Cd}$ & $\mathrm{K}_{\alpha}$ & LiF 200 & Centelleo & 60 & 50 & 15.2918 & -0.33 & 0.49 & 26 & 67 & 30 \\
\hline
\end{tabular}


TABLA 2. COMPOSICIÓN DE LOS PATRONES DE CALIBRACIÓN (\%)

\begin{tabular}{|c|c|c|c|c|c|c|}
\hline \multirow[b]{2}{*}{ Componentes } & \multicolumn{6}{|c|}{ Composición (\%) } \\
\hline & 1 & 2 & 3 & 4 & 5 & 6 \\
\hline $\mathrm{ZrO}_{2}$ & 55.6 & 58.4 & 59.3 & 59.9 & 61.8 & 60.5 \\
\hline $\mathrm{SiO}_{2}$ & 30.6 & 30.1 & 30.3 & 30.5 & 31.4 & 30.8 \\
\hline $\mathrm{Al}_{2} \mathrm{O}_{3}$ & 0.37 & 0.38 & 0.57 & 0.57 & 0.59 & 0.58 \\
\hline $\mathrm{HfO}_{2}$ & 0.77 & 0.81 & 1.20 & 1.21 & 1.24 & 1.22 \\
\hline $\mathrm{TiO}_{2}$ & 0.18 & 0.19 & 0.27 & 0.28 & 0.28 & 0.28 \\
\hline$S$ & 1.53 & 0.76 & 0.96 & 1.14 & 0.66 & 1.10 \\
\hline Se & 1.61 & 0.81 & 0.91 & 1.07 & 0.54 & 0.81 \\
\hline $\mathrm{Cd}$ & 5.72 & 4.83 & 4.68 & 3.90 & 2.23 & 3.58 \\
\hline
\end{tabular}

el apartado anterior para la calibración. La composición de dichos patrones sintéticos se muestra en la tabla 2.

Cada patrón de calibración se preparó por duplicado y se usaron para construir las curvas de calibración. Los datos experimentales de las curvas de calibración se ajustaron minimizando el valor de RMS (Root Mean Square) de acuerdo a la ecuación [1]:

$$
\mathrm{RMS}=\sqrt{\frac{\sum\left(\mathrm{C}^{*}-\mathrm{C}\right)^{2}}{\mathrm{n}-\mathrm{p}}}
$$

Donde $C^{\star}$ es la concentración conocida, C es la concentración calculada, $\mathrm{n}$ es el número de patrones de calibración y p es el número de parámetros calculados de la regresión (pendiente, ordenada en el origen y coeficientes interelementales). En la tabla 3 se muestra el valor de RMS de la calibración para cada uno de los elementos analizados, así como el intervalo de trabajo.

TABLA 3. VALORES DE RMS E INTERVALOS DE TRABAJO EN LA MEDIDA DE CADA ELEMENTO MEDIANTE WD-FRX

\begin{tabular}{|c|c|c|}
\hline $\begin{array}{c}\text { Componentes } \\
(\%)\end{array}$ & RMS & Intervalo de trabajo \\
\hline $\mathrm{ZrO}_{2}$ & 0.17 & $52.56-64.01$ \\
\hline $\mathrm{SiO}_{2}$ & 0.12 & $31.71-37.60$ \\
\hline $\mathrm{Al}_{2} \mathrm{O}_{3}$ & 0.004 & $0.08-1.02$ \\
\hline $\mathrm{HfO}_{2}$ & 0.010 & $1.10-1.29$ \\
\hline $\mathrm{Ti}$ & 0.004 & $0.10-0.29$ \\
\hline $\mathrm{S}$ & 0.20 & $0.54-1.18$ \\
\hline $\mathrm{Cd}$ & 0.016 & $2.52-6.11$ \\
\hline $\mathrm{Se}$ & 0.035 & $0.72-1.45$ \\
\hline
\end{tabular}

Se obtuvieron valores de RMS aceptables para el intervalo de trabajo elegido para todos los elementos a excepción del azufre. El azufre se introdujo en los patrones de calibración sintéticos con los reactivos $\mathrm{SeS}_{2}$ y CdS, ambos sulfuros presentes en los pigmentos, para con ello disponer de patrones sintéticos con matrices similares a las de las muestras a analizar. No se obtuvieron valores de RMS aceptables en la curva de calibrado del azufre debido a la diferente respuesta que cada sulfuro tiene cuando es medido mediante WD-FRX (6), lo cual descartó esta técnica como la apropiada para analizar azufre en este tipo de materiales. Por tanto, el análisis de azufre se llevó a cabo mediante combustión y detección mediante IR.

\subsubsection{Validación de la medida}

Ya que no se dispone de materiales de referencia, se prepararon patrones sintéticos mezclando materiales de referencia y reactivos químicos (distintos a los utilizados en la calibración) en proporciones adecuadas para fabricar patrones de validación con la composición deseada. Se prepararon dos patrones de validación cuya composición se detalla en la tabla 4.

TABLA 4 COMPOSICIÓN DE LOS PATRONES DE VALIDACión ( \%)

\begin{tabular}{|c|c|c|}
\hline \multirow{2}{*}{ Material de referencia (\%) } & \multicolumn{2}{|c|}{ Patrón de Validación } \\
\cline { 2 - 3 } & PATRÓN A & PATRÓN B \\
\hline BCS-CRM 388 Zircon & 92.5 & 94.5 \\
\hline CdS de Aldrich & 1.0 & 2.0 \\
\hline SeS ${ }_{2}$ de Aldrich & 2.5 & 1.5 \\
\hline CdO de Fluka & 4.0 & 2.0 \\
\hline
\end{tabular}

\subsubsection{Medida de las muestras}

Tras la preparación de las curvas de calibrado y su validación, la medida de las muestras a analizar se llevó a cabo en las mismas condiciones que la medida de los patrones de calibración y validación. Se prepararon y midieron al menos tres pastillas de cada muestra para poder calcular la incertidumbre de forma adecuada.

\subsubsection{DETERMINACIÓN DE AZUFRE MEDIANTE COMBUSTIÓN Y DETECCIÓN MEDIANTE IR}

La determinación de azufre se realizó mediante combustión y detección mediante IR utilizando un analizador modelo CS-200 de la firma LECO, provisto de un horno de inducción que alcanza temperaturas de hasta $2000^{\circ} \mathrm{C}$, operando en atmósfera de $\mathrm{O}_{2}$, donde la muestra es introducida y descompuesta por combustión, liberando $\mathrm{SO}_{2}$ que es detectado mediante una celda de IR. Las muestras se pesaron en un crisol de alúmina y se añadió $1 \mathrm{~g}$ de un catalizador (wolframio) para disminuir la temperatura de descomposición y $0.8 \mathrm{~g}$ de un agente que favorece el proceso de inducción (hierro), ya que los pigmentos generalmente no son conductores. 


\subsubsection{Materiales para la calibración y validación}

Para la calibración y validación del análisis de azufre se utilizaron los siguientes materiales de referencia:

a. Materiales de referencia certificados y reactivos químicos para la calibración: SRM 1886a Portland Cement, SRM 1889a Portland Cement y SeS de Alfa Aesar.

b. Materiales de referencia certificados y reactivos químicos usados para la preparación de patrones sintéticos para la validación: BCS-CRM 388 Zircon, CdO de Fluka y SeS 2 de Aldrich.

\subsubsection{Calibración}

La curva de calibrado se construyó con un mínimo de diez puntos, bien pesando distintos pesos del mismo patrón o bien pesando distintos pesos y distintos patrones simultáneamente.

\subsubsection{Validación de la medida}

La validación se llevó a cabo midiendo los patrones sintéticos PATRÓN A y PATRÓN B (preparados a partir de materiales de referencia y patrones químicos distintos a los utilizados en la calibración del método) y comparando los resultados con los valores conocidos.

\subsubsection{Medida de las muestras}

Cada muestra se midió al menos tres veces para poder calcular la incertidumbre adecuadamente. La cantidad de muestra requerida para la determinación depende de la concentración de azufre en la muestra. En muestras con un contenido de azufre inferior al $1 \%$, se encontró que la cantidad de muestra óptima estaba entre 0.2000 y $0.3000 \mathrm{~g}$.

\subsubsection{CÁlCULO DE LA INCERTIDUMBRE DE LA MEDIDA}

La incertidumbre de la medida (8) se calculó como I = $\mathrm{k} \cdot \mathrm{u}_{\text {método' }}$ donde $u_{\text {método }}$ es la incertidumbre combinada calculada a partir de la expresión:

$$
\mathrm{u}_{\text {método }}^{2}=\mathrm{u}_{\mathrm{v}_{\mathrm{R}}}^{2}+\mathrm{u}_{\mathrm{v}_{\mathrm{L}}}^{2}+\mathrm{u}_{\text {REPRO }}^{2}
$$

donde $\mathrm{u}_{\mathrm{V}_{\mathrm{R}}}$ es la incertidumbre del valor certificado del material de referencia $\mathrm{u}_{\mathrm{V}_{\mathrm{L}}}$ es la incertidumbre de la medida del material de referencia y $\mathbf{u}_{\mathrm{REPRO}}$ es la incertidumbre de la medida de la muestra. $\mathrm{u}_{\mathrm{v}_{\mathrm{L}}} \mathrm{y} \mathrm{u}_{\mathrm{REPRO}}$ se calcularon a partir de la expresión $\frac{\mathrm{s}}{\sqrt{\mathrm{n}}}$, donde $s$ es la desviación estándar de la medida del material de referencia o de la medida de la muestra bajo condiciones de reproducibilidad, dependiendo del término calculado, y $n$ es el número de medidas bajo condiciones de reproducibilidad. El factor de covertura $k$ se determina a partir del valor de la t de Student para un $95 \%$ de probabilidad y n-1 grados de libertad.

\section{RESULTADOS Y DISCUSIÓN}

\subsection{Caracterización química mediante WD-FRX}

\subsubsection{RESULTADOS DE LA VALIDACIÓN DE LA MEDIDA}

Para validar las medidas mediante WD-FRX, se midieron los patrones sintéticos PATRÓN A y PATRÓN B. Los resultados obtenidos, junto con su incertidumbre (I) calculada a partir de la expresión [2], se muestran en la tabla 5.

Con objeto de comparar los resultados obtenidos con los valores conocidos de los patrones de validación, se comparó la diferencia entre ambos junto con la incertidumbre relativa, es decir, con la incertidumbre combinada de los valores conocido y medido, tal y como se especifica en la bibliografía $(9,10)$.

El valor absoluto de la diferencia entre el valor medido y el conocido se calcula según la siguiente expresión:

$\Delta_{\mathrm{m}}=\mid \mathrm{c}_{\mathrm{m}}-\mathrm{c}_{\text {conocido }} \mathrm{I}$

donde:

$\Delta_{\mathrm{m}}=$ valor absoluto de la diferencia entre el valor medido y el conocido

$\mathrm{c}_{\mathrm{m}}=$ valor medido

$\mathrm{C}_{\text {conocido }}=$ valor conocido

La incertidumbre de $\Delta_{m}$ se calcula a partir de la incertidumbre del valor conocido y la incertidumbre del valor medido a partir de la siguiente fórmula:

TABLA 5. ResUltAdOS OBTENIDOS EN LA MEDIDA DE LOS PATRONES DE VALIDACIÓN POR WD-FRX

\begin{tabular}{|c|c|c|c|c|}
\hline \multirow{2}{*}{$\begin{array}{c}\text { Componentes } \\
(\%)\end{array}$} & \multicolumn{2}{|c|}{ PATRÓN A } & \multicolumn{2}{c|}{ PATRÓN B } \\
\cline { 2 - 5 } & $\mathbf{C}_{\text {conocida }}$ & $\mathbf{c}_{\text {exp }}$ & C $_{\text {conocida }}$ & $61.0 \pm 0.3$ \\
\hline $\mathrm{ZrO}_{2}$ & $60.0 \pm 0.1$ & $60.3 \pm 0.3$ & $61.3 \pm 0.1$ & $30.8 \pm 0.2$ \\
\hline $\mathrm{SiO}_{2}$ & $30.3 \pm 0.1$ & $30.4 \pm 0.2$ & $30.9 \pm 0.1$ & $0.28 \pm 0.03$ \\
\hline $\mathrm{Al}_{2} \mathrm{O}_{3}$ & $0.27 \pm 0.01$ & $0.25 \pm 0.03$ & $0.27 \pm 0.03$ & $1.22 \pm 0.02$ \\
\hline $\mathrm{HfO}_{2}$ & $1.20 \pm 0.08$ & $1.21 \pm 0.02$ & $1.23 \pm 0.08$ & $0.23 \pm 0.02$ \\
\hline $\mathrm{TiO}_{2}$ & $0.21 \pm 0.01$ & $0.20 \pm 0.02$ & $0.22 \pm 0.01$ & $0.84 \pm 0.02$ \\
\hline $\mathrm{Se}$ & $1.34 \pm 0.01^{\text {(a) }}$ & $1.32 \pm 0.02$ & $0.81 \pm 0.01^{(\mathrm{a})}$ & $3.62 \pm 0.04$ \\
\hline $\mathrm{Cd}$ & $4.41 \pm 0.01^{\text {(a) }}$ & $4.41 \pm 0.04$ & $3.58 \pm 0.01^{\text {(a) }}$ & \\
\hline
\end{tabular}

(a) El cálculo de la incertidumbre está basado en el número de cifras significativas que aparecen en el certificado- 
TABLA 6. COMPARACIÓN DE LOS RESUlTAdOS DE LOS PATRONES DE VALIDACIÓN CON EL VALOR CONOCIDO

\begin{tabular}{|c|c|c|c|c|}
\hline \multirow{2}{*}{$\begin{array}{c}\text { Componentes } \\
(\%)\end{array}$} & \multicolumn{2}{|c|}{ PATRÓN A } & \multicolumn{2}{|c|}{ PATRÓN B } \\
\hline & $\Delta_{\mathrm{m}}$ & $I_{\Delta m}$ & $\Delta_{\mathrm{m}}$ & $\mathbf{I}_{\Delta \mathrm{m}}$ \\
\hline $\mathrm{ZrO}_{2}$ & 0.3 & 0.6 & 0.3 & 0.6 \\
\hline $\mathrm{SiO}_{2}$ & 0.3 & 0.4 & 0.1 & 0.4 \\
\hline $\mathrm{Al}_{2} \mathrm{O}_{3}$ & 0.02 & 0.06 & 0.01 & 0.06 \\
\hline $\mathrm{HfO}_{2}$ & 0.01 & 0.17 & 0.01 & 0.17 \\
\hline $\mathrm{TiO}_{2}$ & 0.01 & 0.05 & 0.01 & 0.05 \\
\hline Se & 0.02 & 0.05 & 0.03 & 0.05 \\
\hline
\end{tabular}

TABLA 7. RESULTADOS OBTENIDOS EN EL ANÁLISIS DE LOS ELEMENTOS MAYORITARIOS Y MINORITARIOS MEDIANTE WD-FRX PARA LOS CINCO PIGMENTOS ANALIZADOS

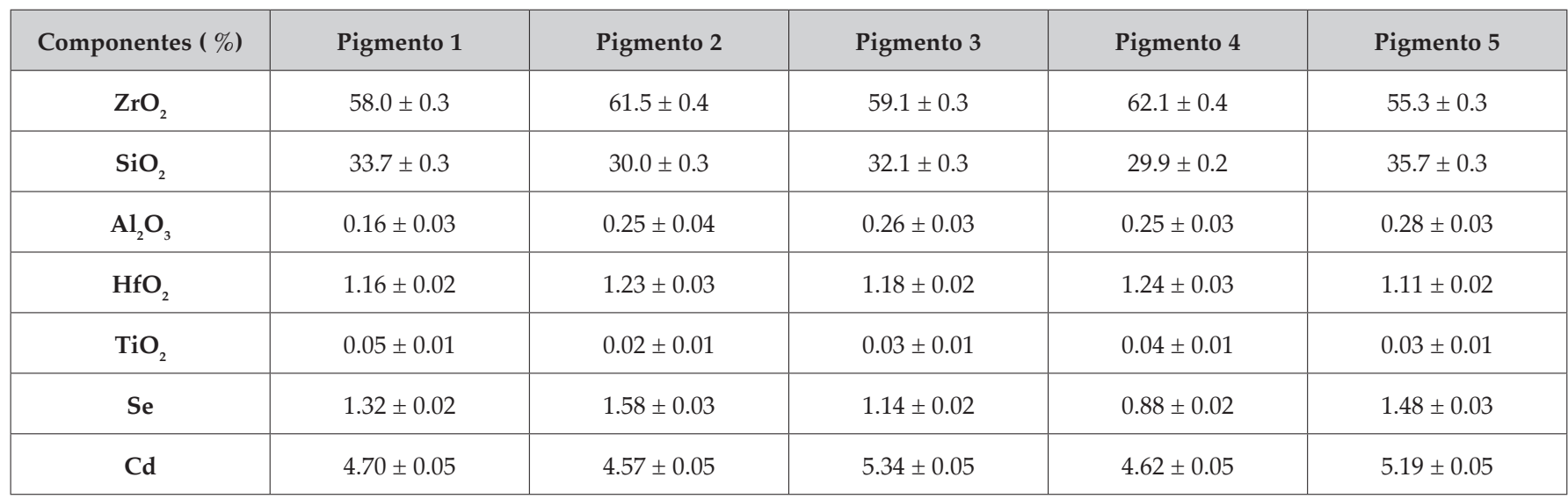

$$
\mathrm{u}_{\Delta \mathrm{m}}=\sqrt{\mathrm{u}_{\mathrm{m}}^{2}+\mathrm{u}_{\text {conocido }}^{2}}
$$

donde:

$\mathrm{u}_{\Delta \mathrm{m}}=$ incertidumbre combinada del resultado y el valor conocido

$\mathrm{u}_{\mathrm{m}}=$ incertidumbre del valor medido

$\mathrm{u}_{\text {conocido }}=$ incertidumbre del valor conocido

La incertidumbre expandida $I_{\text {s }}$ se obtiene multiplicando $u_{\Delta m}$ por un factor de cobertura $(\mathrm{k})$, normalmente igual a dos, lo cual corresponde aproximadamente a un $95 \%$ de nivel de confianza. Por tanto:

$$
\mathrm{I}_{\Delta \mathrm{m}}=2 \cdot \mathrm{u}_{\Delta \mathrm{m}}
$$

Para verificar la bondad del método, $\Delta_{m}$ se compara con $\mathrm{I}_{\Delta m^{\prime}}$ de modo que si $\Delta_{\mathrm{m}} \leq \mathrm{I}_{\Delta \mathrm{m}^{\prime}}$, no hay diferencias significativas entre el valor medido y el valor conocido, quedando validado así el método.

Los resultados de esta comparación se muestran en la tabla 6.

Los resultados obtenidos muestran que se cumple $\Delta_{\mathrm{m}} \leq \mathrm{I}_{\Delta \mathrm{m}^{\prime}}$ por lo que no existen diferencias significativas, lo cual valida el método desarrollado tanto para los elementos mayoritarios como para los minoritarios.

\subsubsection{RESULTADOS DEL ANÁLISIS DE LOS PIGMENTOS A BASE DE SULFOSELENIURO DE CADMIO}

En la tabla 7 se muestran los resultados obtenidos en el análisis mediante WD-FRX junto con su incertidumbre (I), calculada a partir de la expresión [2].

Cabe destacar las bajas incertidumbres obtenidas para todos los elementos analizados con la metodología propuesta.

\subsection{Determinación de azufre mediante combustión y detección mediante IR}

\subsubsection{RESULTADOS DE LA VALIDACIÓN DE LA MEDIDA}

En la tabla 8 se muestran los resultados de la validación de la medida de azufre junto con su incertidumbre, calculada a partir de la expresión [2].

TABLA 8. RESULTADOS DE LA MEDIDA DE AZUFRE POR COMBUSTIÓN Y DETECCIÓN MEDIANTE IR DE LOS PATRONES DE VALIDACIÓN

\begin{tabular}{|c|c|c|}
\hline \multirow{2}{*}{$\begin{array}{c}\text { Patrón de } \\
\text { Validación }\end{array}$} & \multicolumn{2}{|c|}{$\mathbf{S}(\%)$} \\
\cline { 2 - 3 } & Valor conocido & Valor obtenido \\
\hline PATRÓN A & $1.31 \pm 0.01^{\text {(b) }}$ & $1.28 \pm 0.05$ \\
\hline PATRÓN B & $1.10 \pm 0.01^{\text {(b) }}$ & $1.12 \pm 0.05$ \\
\hline
\end{tabular}

(b) El cálculo de la incertidumbre está basado en el número de cifras significativas que aparecen en el certificado de análisis 
Con objeto de comparar los resultados obtenidos con los valores certificados o conocidos en los patrones de validación, se utilizó el procedimiento descrito anteriormente para los elementos analizados mediante WD-FRX. En la tabla 9 se muestran los resultados de esta comparación.

Tabla 9. Comparación de los Resultados de la medida de azufre POR COMBUSTIÓN Y DETECCIÓN MEDIANTE IR DE LOS PATRONES DE VALIDACIÓN

\begin{tabular}{|c|c|c|}
\hline $\begin{array}{c}\text { Patrón de } \\
\text { Validación }\end{array}$ & $\boldsymbol{\Delta}_{\mathrm{m}}$ & $\mathbf{I}_{\Delta \mathrm{m}}$ \\
\hline PATRÓN A & 0.03 & 0.10 \\
\hline PATRÓN B & 0.02 & 0.10 \\
\hline
\end{tabular}

Los resultados obtenidos para los patrones de validación muestran que $\Delta_{\mathrm{m}} \leq \mathrm{I}_{\Delta \mathrm{m}^{\prime}}$ por lo que no existen diferencias significativas entre el valor conocido y el valor obtenido, estando el método validado.

\subsubsection{RESULTADOS DEL ANÁLISIS DE LOS PIGMENTOS A BASE DE SULFOSELENIURO DE CADMIO}

En la tabla 10 se presentan los resultados obtenidos en el análisis de azufre en los cinco pigmentos analizados, junto con su incertidumbre (I), calculada a partir de la expresión [2].

Las incertidumbres obtenidas para todos los pigmentos son muy pequeñas, lo cual hace que la metodología sea precisa y exacta.

\section{CONCLUSIONES}

1. Se ha desarrollado un método para la caracterización química completa de pigmentos cerámicos a base de sulfoseleniuro de cadmio, utilizando WD-FRX y análisis mediante combustión y detección mediante IR, donde todos los elementos pueden ser analizados mediante WD-FRX excepto el azufre que tiene que ser analizado mediante otros métodos tales como el análisis mediante combustión y detección mediante IR. Mediante este método desarrollado es la primera vez que se dispone de un método cuantitativo para todos los elementos presentes en este tipo de muestras, incluido el selenio y el azufre.

2. La ausencia de materiales de referencia certificados con una composición similar a los pigmentos a analizar hace necesaria la preparación de patrones sintéticos mezclando los materiales de referencia de que se dispone y reactivos químicos.
3. El análisis mediante WD-FRX tiene que llevarse a cabo preparando las muestras en forma de pastillas prensadas ya que este tipo de materiales presenta elementos volátiles tales como el azufre y el selenio, lo cual hace imposible el uso de perlas, donde la temperatura de procesado del material es elevada.

4. La metodología desarrollada permite la completa caracterización de estos pigmentos cerámicos, obteniendo resultados con incertidumbres muy bajas para todos los elementos analizados, lo cual hace que el método sea preciso y exacto.

\section{AGRADECIMIENTOS}

Este trabajo ha sido financiado por el Instituto Valenciano de Competitividad Empresarial (IVACE) dentro del programa de Investigación, Desarrollo e Innovación, a través del proyecto IMDEEA/2012/139, a través del Fondo Europeo de Desarrollo Regional de la Unión Europea.

\section{BIBLIOGRAFÍA}

1. A. BROLL, H. MANN, Inorganic coloured pigments and a process for their production. GB 1440718. 1976-06-23.

2. H. B. WAGNER; A. BROLL, Process for the production of firing stable yellow to red glaze mixtures. US 4008091. 1977-02-15.

3. M LLUSAR, Optimización medioambiental de la síntesis de pigmentos cerámicos: aplicación de métodos sol-gel. Castellón: Universitat Jaume I, 1998. Tesis doctoral.

4. J. BELTRÁN, C. GUILLEN, V. LABIES, Estudio de la formación de pigmento cerámico de sulfoseleniuro de cadmio a partir de CdS y Se. II. Estudio cinético. Bol. Soc. Esp. Ceram. Vidr., 291-294, 16 (5), 1977.

5. Y. ZHANG, J.C. DENG, X.D. GU, New method synthesizes scarlet pigments at low temperature. Am. Ceram. Soc. Bull., 9301-9305, 85 (3), 2006.

6. M.F. GAZULLA, M.P. GÓMEZ, M. ORDUÑA, M. RODRIGO, New methodololy for sulfur analysis in geological samples by WD-XRF spectrometry. X-ray Spectrometry, 3-8, 38, 2009.

7. EN 15309:2007 Characterisation of waste and soil. Determination of elemental composition by X-ray fluorescence. 2007.

8. Use of Reference Materials and the Estimation of Measurement Uncertainty. Joint Research Center, Brussels, October 15-16, 2008. [Training course].

9. M.F. GAZULLA, M. RODRIGO, S. VICENTE, M. ORDUÑA, Methodology for the determination of minor and trace elements in petroleum cokes. X-ray Spectrometry 321-327, 39 (5): 2010.

10. T. LISINGER. Comparison of a Measurement Result with the Certified Value. European Communities, Geel 2005. http://www.erm-crm.org/html/ERM products/application_notes /application_note_1/application_note_1_english_ en.pdf [Accesed: 2009-04-24]

Recibido: $12 / 07 / 2013$

Recibida versión corregida: $21 / 10 / 2013$

Aceptado: 28/10/2013

TABla 10. Resultados del ANÁlisis de AZUFRE POR COMBUSTIÓN y DETECCión MEDiANTE IR

\begin{tabular}{|c|c|c|c|c|c|}
\hline & Pigmento 1 & Pigmento 2 & Pigmento 3 & Pigmento 4 \\
\hline$S(\%)$ & $0.99 \pm 0.05$ & $1.05 \pm 0.04$ & $0.87 \pm 0.05$ & $1.00 \pm 0.05$ \\
\hline
\end{tabular}

